\title{
La Restauration de la musique de la Chapelle royale et les fantômes de l'Ancien Régime (1814-1815)
}

The Restoration of the Music of the Royal Chapel and the Ghosts of the Old Regime (1814-1815)

\section{Youri Carbonnier}

\section{OpenEdition}

Journals

\section{Édition électronique}

URL : https://journals.openedition.org/ahrf/13443

DOI : $10.4000 /$ ahrf. 13443

ISSN : 1952-403X

Éditeur :

Armand Colin, Société des études robespierristes

\section{Édition imprimée}

Date de publication : 15 février 2015

Pagination : 165-182

ISBN : 978-2-200-92958-9

ISSN : 0003-4436

\section{Référence électronique}

Youri Carbonnier, « La Restauration de la musique de la Chapelle royale et les fantômes de l'Ancien Régime (1814-1815) », Annales historiques de la Révolution française [En ligne], 379 | janvier-mars 2015 mis en ligne le 15 février 2018, consulté le 01 juillet 2021. URL : http://journals.openedition.org/ahrf/ 13443 ; DOI : https://doi.org/10.4000/ahrf.13443 


\section{LA RESTAURATION DE LA MUSIQUE DE LA CHAPELLE ROYALE ET LES FANTÔMES DE L'ANCIEN RÉGIME (1814-1815)}

Youri CARBONNIER

Lors de la première Restauration, en 1814, la Musique impériale est provisoirement maintenue sous la direction de Jean-François Le Sueur, malgré les réclamations d'anciens musiciens du roi survivants. La nouvelle Musique du roi, organisée en janvier 1815, garde l'essentiel des acquis de la période précédente, dont son directeur, et la moitié de son effectif. Elle intègre quelques anciens, dont le surintendant Martini, mais aussi bon nombre de nouveaux venus. L'Ancien Régime transparaît dans des choix terminologiques et dans le remplacement des femmes par des jeunes garçons. L'essai avorté de revenir en arrière durant les Cent-Jours entraîne toutefois le retour des chanteuses et donne à la Musique du roi la physionomie qu'elle garde jusqu'à sa dissolution en 1830. Elle s'inscrit moins dans un impossible retour à l'Ancien Régime que dans la continuité de la Chapelle impériale, elle-même inspirée de celle des anciens rois.

Mots-clés : Musique du roi, Chapelle, musique, Ancien Régime, Restauration

Jusqu'au 10 août 1792, la Musique du roi constitue un rouage essentiel, car quotidien, de la vie de la Cour, déjà diminué après le transfert aux Tuileries $^{1}$, puis nettement affaibli après l'incident de Varennes, entraînant le

(1) Sur les conditions de travail des musiciens et l'aménagement de la chapelle des Tuileries, voir Youri CARBONNIER, « Les Musiciens du roi face à la Révolution », Revue de l'histoire de Versailles et des Yvelines, t. 90, 2008, p. 20-23. 
déplacement de la messe dans la galerie des Ambassadeurs, vraisemblablement sans musique ${ }^{2}$. Depuis le règne de Louis XIV, plus particulièrement depuis l'installation à Versailles, la messe basse quotidienne, accompagnée de musique, est en effet un moment phare, durant lequel chaque courtisan peut voir le roi et en être vu, ce qui en fait un moyen de gouvernement et d'éclat de la souveraineté.

C'est à ce titre que la messe en musique, désormais dominicale ${ }^{3}$, fait sa réapparition en 1802, gagnant progressivement en pompe avec l'augmentation des effectifs musicaux entamée en $1804^{4}$, puis avec l'aménagement, par Percier et Fontaine, de la chapelle des Tuileries, inaugurée le 2 février 1806. L'aménagement de la tribune de la Musique y doit beaucoup à l'inspiration versaillaise, ce qui n'a pas été souligné jusqu'à présent : face à la tribune du souverain, elle accueille les musiciens dans un espace en amphithéâtre aménagé au-dessus de la sacristie et surplombant l'autel ${ }^{5}$. Le mimétisme va jusqu'au maintien du partage des exécutants en deux groupes, à droite et à gauche, pratique courante à la chapelle de Versailles ${ }^{6}$. La Musique de la Chapelle du roi ne peut échapper à la vaste opération de restauration de la Cour d'Ancien Régime mise en œuvre

(2) Hubert Robert nous en a laissé une image saisissante dans La dernière messe de la famille royale aux Tuileries, 1792 (coll. part.). Courte analyse dans François PUPIL, « Les fidèles à l'église dans la peinture française ", Revue de l'histoire des religions, 2000, vol. 217, n 3, p. 403 ; reproduction dans Olivier MiCHEL, " "Avoir tout ce qu'il y a de beau en Italie" ou quelques avatars de la Galerie des Carrache », dans Les Carrache et les décors profanes, Rome, École française de Rome, 1988, p. 477-490:479. La présence de musique est toutefois attestée par le témoignage de Mme Campan sur les vêpres du dernier dimanche avant la fin de la monarchie, durant lesquelles les musiciens firent preuve d'un esprit frondeur en enflant leurs voix pour le Deposuit potentes de sede du Magnificat (Mémoires de Madame Campan, Paris, Mercure de France, 1988, p. 322). Il est possible que certains offices aient pris place à la chapelle, comme semble l'indiquer le journal de Louis XVI pour le 4 août 1791 (Mathieu CouTy, La vie aux Tuileries pendant la Révolution, 1789-1799, Paris, Tallandier, 1988, p. 132).

(3) Mais le jeudi accueille également un office religieux (Pierre BRANDA, Napoléon et ses hommes. La Maison de l'Empereur 1804-1815, Paris, Fayard, 2011, p. 184 et 201).

(4) Sur cette réorganisation, qui coïncide avec la nomination de Le Sueur à la tête de la Musique : Jean MONGRÉDIEN, Jean-François Le Sueur, contribution à l'étude d'un demi-siècle de musique française (1780-1830), Berne, Peter Lang, 1980, p. 803-828.

(5) Louis HAUTECEUR, Histoire de l'Architecture classique en France, t. V, Paris, Picard, 1953, p. 164-166 ; Jean MONGRÉDIEN, Jean-François Le Sueur, op. cit., p. 808-810 ; Guillaume FONKENELL, Le Palais des Tuileries, Arles / Paris, Honoré Clair / Cité de l'architecture et du patrimoine, 2010, p. 145-147. À la différence de Versailles, la Musique semble au moins partiellement cachée aux regards « derrière des grilles voilées » (selon Le Sueur, dans sa « Lettre sur la Musique du roi Louis XVIII », S.I.M., VII ${ }^{\mathrm{e}}$ année, $\mathrm{n}^{\circ} 10$, octobre 1911, p. 4, mais le sens exact du passage, à propos de la présence de chanteuses, n'est pas clair), élément confirmé par des images du Second empire, mais absent des gravures antérieures.

(6) Disposition bien visible sur le plan de Métoyen : BM Versailles, Ms F 87, Plans des Tribunes \& Orchestres de la Musique du Roy, Avec les Noms des Sujets qui en occupent les Places. En l'Année 1773. Plusieurs listes des musiciens de la période impériale distinguent, dans un même pupitre, ceux « de la droite » de ceux « de la gauche» $\left(\mathrm{AN}, \mathrm{O}^{2} 62\right)$. 
en 1814. Impliquant des compétences particulières, la Musique est peu concernée par la course aux places qui, au sein de la Maison du roi, oppose les rejetons de l'ancienne noblesse à ceux de la noblesse impériale ${ }^{7}$. Pour autant, elle n'échappe pas tout à fait à la brigue des anciens musiciens ou aux réclamations de leurs descendants, avec un succès relatif et variable selon la période.

\section{Parer au plus pressé : la Musique provisoire de 1814}

Dans les journées confuses d'avril 1814, rares sans doute sont ceux qui se soucient de la situation de la Musique de la Chapelle. Le directeur de la Musique impériale, Jean-François Le Sueur (notre unique source directe, et combien prolixe, pour cette période), se présente aussitôt comme l'homme de la situation, capable d'offrir à Louis XVIII - et en attendant, à son frère le comte d'Artois, entré à Paris le 12 avril avec le titre de lieutenant général du royaume et aussitôt assailli « de placets, de pétitions, de mémoires et d'écrits $\gg^{8}-$ un ensemble musical digne de la monarchie des Bourbons restaurée. Il se démène pour le maintien du statu quo, obtenu provisoirement le $1^{\mathrm{er}}$ mai, et pour donner au roi un accueil digne de Louis le Désiré : le Te Deum célébré à Notre-Dame le 3 mai doit constituer la preuve de l'opportunité de sa proposition, face aux contre-projets qui se multiplient ${ }^{9}$.

Le 16 mai 1814, il adresse au comte de Blacas, ministre de la Maison du roi, et au duc de Duras, premier gentilhomme de la Chambre $^{10}$, un mémoire extrêmement précis sur la Musique provisoire, assorti de comparaisons avec celle de l'Ancien Régime et d'une justification détaillée de ses choix pour l'avenir, documents qui lui auraient été demandés « par écrit, dès le mois d'avril » et qu'il signe « le chevalier Le Sueur, provisoirement directeur et compositeur de la musique du Roi ${ }^{11}$.

(7) Francis DÉMIER, La France de la Restauration (1814-1830), Paris, Gallimard, 2012, p. 68-69, 81 ; Emmanuel DE WARESQUIEL, Benoît YVERT, Histoire de la Restauration 1814-1830, Paris, Perrin, 2002, p. 72-93 ; Philip MANSEL, La Cour sous la Révolution, l'exil et la Restauration 1789-1830, Paris, Tallandier, 1989, p. 115-131 ; Id., Louis XVIII, Paris, Pygmalion, 1982, p. 218-219.

(8) Pierre François Léonard FonTAINE, Journal. 1799-1853, Paris, École nationale supérieure des Beaux-Arts / Institut français d'architecture / Société de l'histoire de l'art français, 1987, vol. 1, p. 411.

(9) Sur ces premiers jours et les prétentions, en particulier de Choron, à organiser et diriger la cérémonie de Notre-Dame : Jean MongRÉDIEN, Jean-François Le Sueur, op. cit., p. 831-832. grand-père.

(10) Le duc de Duras retrouve cette charge qu'il occupait déjà en 1789 , à la suite de son

(11) AN, $\mathrm{O}^{3} 375$, Direction Provisoire de la Musique du Roi, conservée par ordonnance de S.A.R. Monsieur, Lieutenant General du Royaume, du $1^{\text {er }}$ mai 1814 [désormais Direction Provisoire] ; il s'agit d'un duplicata, complété d'une longue note autographe de Le Sueur, datable d'août 1814. 
La comparaison du dernier état de paiement des musiciens de l'Empire ${ }^{12}$ avec celui que fournit Le Sueur atteste une continuité quasi absolue, soulignée par un papillon sur le titre de ce dernier, où une main anonyme a tracé : «État de la Musique de la chapelle de Buonaparte », suivi de, au crayon, «telle qu'elle existe encore». On ne saurait plus clairement définir la stabilité de la situation, assez semblable à celle des autres départements de la Maison du roi, en particulier des services techniques ${ }^{13}$.

À y regarder de plus près, l'effectif monte à cent-six personnes (dont cent-un musiciens) au lieu de quatre-vingt dix-sept (quatre-vingt onze musiciens) : quatre artistes absents et quatorze nouveaux. Tout à la défense de l'ensemble qu'il dirige depuis une décennie, Le Sueur bâtit ainsi habilement l'image d'une machine solide, parfaitement opérationnelle. Trois chanteuses sont absentes, «vu des rapports faits contre elles, mais qui paroissent être erronés $»^{14}$, ainsi que le hautboïste Gebauer, disparu pendant la retraite de Russie à la tête de la musique de la Garde impériale ${ }^{15}$. Les nouveaux sont soit des chanteurs (quatre femmes et sept ténors ${ }^{16}$ ), soit des membres d'un «quatuor récitant» (deux harpes, cor et violoncelle) ${ }^{17}$. Par cette augmentation d'effectif, Le Sueur fige la masse salariale à un niveau élevé, tout en s'ingéniant à démontrer l'économie réalisée par rapport à 1789. Malgré cela, pour deux tiers des nouveaux ce ne sera qu'un feu de paille, qu'illustre le refus de paiement signifié au ténor

Les mêmes arguments sont développés dans une lettre adressée à Mornard, secrétaire de Talleyrand, mais surtout, secrétaire de la Musique dans la dernière décennie de l'Ancien Régime : « Lettre sur la Musique... », op. cit., p. 1-7 (p. 1 pour la mention d'une demande écrite en avril).

(12) AN, $\mathrm{O}^{2}$ 62, Exercice an 1814, mois de mars, Maison de S. M. l'Empereur et Roi. Service du Grand Chambellan.

(13) Philip MAnsel, La Cour... op. cit., p. 120. Le 4 mai, Fontaine écrit qu' « on a conservé jusqu'ici presque toutes les personnes qui sont restées à leur poste » (Pierre François Léonard FONTAINE, op. cit., p. 415). Sur la stabilité des administrations, Emmanuel DE WARESQUIEL, Benoît YVERT, op. cit., p. 69-71.

(14) $\mathrm{AN}, \mathrm{O}^{3} 375$, Direction provisoire. L'une d'elles, Mme de Staiti, avait été autorisée à aller en Italie Ibid., $n^{\circ} 9$, réclamation du 11 janvier 1815).

(15) D'abord signalé « prisonnier en Russie » (AN, $\mathrm{O}^{1} 842, \mathrm{n}^{\circ} 104$, ajout marginal), puis «cru décédé en russie » et remplacé par Chol, en janvier $1815\left(\mathrm{O}^{3} 375\right.$, liste sans titre), il est enfin déclaré mort « à Wilna - $9^{\text {bre }} 1812$ » (ibid., État des Musiciens de la Chapelle décédés en exercice, s.d. [1826]). Cette situation empêche sa veuve d'obtenir une pension $\left(\mathrm{O}^{3 *} 279, \mathrm{p} .47, \mathrm{n}^{\circ} 170\right.$, lettre du 11 décembre 1815 à Mme Gebauer). insuffisant.

(16) Surtout des ténors choristes : ils ne sont que deux en mars 1814, ce qui est effectivement

(17) Le Sueur ajoute un « $2^{\mathrm{e}}$ harpiste », Nadermann cadet, et le violoncelliste Duport, qui rejoignent Nadermann l'aîné et le corniste Frédéric Duvernoy. Cet ensemble était dédié aux petits concerts chez l'empereur (Victorine DE CHASTENAY, Mémoires de Madame de Chastenay 1771-1815, t. 2, Paris, Plon, 1897, p. 130), tout en étant sollicité à la chapelle dès 1802 (Jean MonGRÉDIEN, La musique en France des Lumières au Romantisme 1789-1830, Paris, Flammarion, 1986, p. 168-169). 
Ménars en 1815, car il n'apparaît que sur un projet et n'a été convoqué que «très irrégulièrement ${ }^{18}$. Enfin, l'organiste Nicolas Séjan semble avoir été ajouté après coup, pour réparer un oubli bien compréhensible du fait que la chapelle des Tuileries ne possédait pas d'orgue à cette époque ${ }^{19}$. De fait, le grand aumônier de Louis XVIII, Talleyrand-Périgord, a chargé Séjan de tenir les claviers, dès qu'il aurait trouvé un instrument. Aidé du facteur Dallery, Séjan fait transférer en urgence l'orgue de la chapelle du château de Saint-Cloud, qui sonne aux Tuileries dès le dimanche 8 mai $^{20}$.

Dans ce document essentiel, Le Sueur tâche avant tout de pérenniser la situation provisoire de la Musique. Pour cela, il avance en particulier deux arguments : son efficacité, forgée par une longue pratique commune qui, seule, permet « la précision vocale exigée ${ }^{21}$, et l'économie réalisée. C'est là l'argument suprême, asséné dès la première page : même en ajoutant aux musiciens en place huit ou neuf anciens encore en état de servir, le budget global (180 000 francs) demeure inférieur aux 260000 francs prévus par l'édit portant règlement sur la Musique du roi de mai 1782. Cette économie est due au fait que les artistes actuels, les meilleurs musiciens de Paris, peuvent se contenter de traitements moindres, car ils sont aussi salariés d'un des théâtres de la capitale, ce que les astreintes d'un service quotidien à Versailles rendaient impossible sous l'Ancien Régime. Ce faisant, Le Sueur fourbit ses armes pour sauvegarder les titulaires, face à une offensive des anciens musiciens du roi, qui explique à la fois les huit à neuf places offertes à ces derniers dans son projet et la mention entre parenthèses « de l'ancienne $\mathrm{M}^{\text {que }}$ du Roi », pour cinq artistes en exercice ${ }^{22}$.

(18) $\mathrm{AN}, \mathrm{O}^{3 *} 279$, p. $23, \mathrm{n}^{\circ} 85$, lettre du 15 octobre 1815 .

(19) Par la suite, Dallery construit un premier instrument en 1819 (AN, O3 221 ; autre exemplaire publié par Norbert DUFOURCQ, Autour des orgues du Conservatoire national et de la chapelle des Tuileries, Paris, Floury, 1952, tiré à part de la revue L'Orgue, ${ }^{\circ}$ 58-62, 1951-1952).

(20) Toute l'affaire est racontée par Séjan lui-même dans un courrier réclamant le paiement de son service à la Chapelle de mai à décembre 1814. Ayant été appelé par le grand aumônier, il n'a pas été porté sur les états de paiement de la Musique avant son organisation définitive, le $1^{\mathrm{er}}$ janvier 1815 , et a donc été oublié ( $\left.\mathrm{AN}, \mathrm{O}^{3} 282\right)$.

(21) En réponse à la proposition d'engager des élèves du Conservatoire, Le Sueur ne craint pas d'affirmer qu' " il leur faudrait dix ans d'expérience musicale » pour rendre correctement le répertoire de la Chapelle, constitué de «trente messes, les meilleures de Paisiello et du Directeur actuel ». La ficelle est un peu grosse, surtout pour un homme qui a justement fait chanter ces élèves durant les premières années de l'Empire, mais il est vrai qu'il s'adresse à des béotiens en matière de musique, dont l'adhésion peut facilement être emportée par l'orgueilleuse assurance d'un spécialiste reconnu. Ailleurs, Le Sueur retourne l'argument, expliquant l'audience favorable que reçoivent les contre-projets par le fait que les décideurs sont insuffisamment éclairés « sur une matière qu'on ne connoit point » (« Lettre sur la Musique... », op. cit., p. 3).

(22) La Musique compte en fait sept anciens membres de la chapelle de Louis XVI, dont le fameux violoniste Rodolphe Kreutzer, dédicataire d'une sonate de Beethoven, entré en 1785. 
Cette offensive des « revenants $»^{23}$ est-elle réelle ? Est-elle aussi massive et dangereuse que Le Sueur le prétend ? Ne s'agit-il pas pour le directeur de la Musique de sauver avant tout sa propre position?

\section{L'offensive des « revenants » de l'Ancien Régime}

Dans son long mémoire, Le Sueur évoque à plusieurs reprises une offensive des anciens musiciens du roi visant, selon lui, à évincer et à remplacer les artistes en place. Elle s'inscrit dans le mouvement de reconstitution informelle de la cour, encouragé par la volonté royale « de rappeler de préférence à tous autres les anciens titulaires $»^{24}$.

Sans jamais nommer ses adversaires, Le Sueur concentre ses piques sur un « survivancier-surintendant » qu'il est aisé d'identifier. En effet, Jean Paul Gilles Martini ${ }^{25}$ avait obtenu la survivance d'une charge de surintendant de la Musique du roi le 28 février $1788^{26}$. À ce titre, comme les autres anciens titulaires d'offices ou héritiers d'officiers de l'ancienne monarchie, Martini peut réclamer la fonction ou, à défaut, le remboursement de la finance de sa charge, que l'état du Trésor ne permet pas en fait d'envisager ${ }^{27}$. Selon Le Sueur, son rival « a prouvé un très beau talent par plusieurs ouvrages au théâtre de l'opera comique, mais [il] n'a jamais été maître de chapelle » et il est vrai que la tâche du surintendant était presque exclusivement profane avant la Révolution ${ }^{28}$. Ce compositeur (« non français », précise-t-il fielleusement) n’aurait de toute façon « point

(23) Le terme, tout à fait adapté à la situation de certains anciens musiciens du roi, disparus du paysage musical depuis 1792, est employé par Fontaine dans son journal pour désigner ses concurrents surgis de l'Ancien Régime : Pierre François Léonard FONTAINE, op. cit., p. 416.

(24) Philip MANSEL, La Cour...op. cit., p. 113 et p. 115-116 pour la citation.

(25) Né en 1741 à Freystadt, en Bavière, Johann Paul Aegidius Martin dit Schwarzendorf avait italianisé son nom en « Martini il Tedesco », à son arrivée en Lorraine vers 1760. En France, Aegidius est traduit par Gilles, auquel les dictionnaires préfèrent Égide (dès Alexandre CHORON, François FAYOLLE, Dictionnaire historique des musiciens, Paris, Chimot, 1817, t. II, p. 26).

(26) $\mathrm{AN}, \mathrm{O}^{1} 676, \mathrm{n}^{\circ} 500,501$ et 503 , dossier de François Giroust, titulaire de la charge. La survivance est, pour le survivancier, l'assurance d'entrer en charge au décès ou à la démission du titulaire de l'office.

(27) Thibaut TRÉTOUT, « La transmission héréditaire des charges de la Maison civile du roi sous la Restauration. Le cas des maîtres de l'hôtel (1814-1815) », Hypothèses, 2009/1, n 12, p. 67-73 : 63-64. Martini affirme avoir déboursé 25000 francs pour cette charge, dont 16000 versés à Giroust (BNF, Musique, Lettres autographes, vol. 71, 213 bis, lettre au Directeur Reubell).

(28) À l'actif de Martini, dont la postérité retient surtout la romance « Plaisir d'amour », citons néanmoins une messe solennelle, publiée en 1808, et un Te Deum donné en 1809, ou la publication d'une École d'orgue [...] d'après les ouvrages des plus célèbres organistes de l'Allemagne, et dédiée à Sa Majesté l'impératrice Joséphine, Paris, Imbault, s.d. [vers 1805], fruit de son expérience précoce d'organiste. 
l'intention, vu son grand âge, de faire les services actifs, ni de composer les musiques nécessaires à la chapelle, ce qui est un travail considérable $»^{29}$.

Pour Le Sueur, le vieux survivancier incarne surtout la menace de perdre une position confortable et enviable, longtemps attendue, qu'il considère avoir obtenue par son seul mérite ${ }^{30}$. Cela suffit sans doute à expliquer l'acharnement presque obsessionnel avec lequel il dénonce son rival dans ses écrits de l'été 1814. Martini est-il le Machiavel qu'il décrit ? Sans doute pas. Certes, dans une lettre adressée le 20 avril 1814 au comte Beugnot, ministre de l'Intérieur, Martini s'appuie sur le rôle dévolu au surintendant pour obtenir l'exécution de son Te Deum à Notre-Dame lors de l'entrée de Louis XVIII, mais, comme Le Sueur, il porte ses coups contre Choron $^{31}$. S'il est certain que la musique de Martini se fait entendre à la chapelle ${ }^{32}$, les traces de son action sont rares dans les archives. Mais son influence, potentiellement réelle (il dirigeait en 1789 l'orchestre du théâtre de Monsieur, placé sous la protection du futur Louis XVIII ${ }^{33}$ ), a pu user d'intermédiaires. Ainsi, fut-il peut-être à l'origine des listes d'anciens musiciens qui ont fleuri alors ; il semble en tout cas avoir promis, hors de tout cadre formel, des emplois à certains ${ }^{34}$.

Quoi qu'il en soit, Martini n'agit pas seul dans ce retour des anciens et Le Sueur évoque « plusieurs musiciens de la Chapelle de Louis XVI », voire « quelques anciens pensionnaires de la musique de Louis XV » qui

(29) Toutes les citations sont tirées de AN, $\mathrm{O}^{3}$ 375, Direction Provisoire. Les mêmes idées, assorties de critiques plus acerbes contre Martini, sont développées dans la « Lettre sur la Musique... », op. cit., passim. Martini a néanmoins composé pendant son service un Requiem pour la messe obituaire de Louis XVI, huit messes et sept élévations (BNF, Musique). Par ailleurs, si la signature tremblée de Martini accuse sa faiblesse en 1815, ce n'est pas encore le cas en 1814.

(30) Après des débuts précoces et prometteurs, Le Sueur avait échoué, malgré l'appui de la reine et de nombreuses recommandations, qu'il sut opportunément ressortir en 1814 (AN, $\mathrm{O}^{3} 245$ ), à obtenir une place de maître de musique de la Chapelle royale en 1786. Agé de 26 ans, joué au Concert spirituel, il avait alors obtenu la direction de la musique de Notre-Dame de Paris dont il fut écarté dès l'année suivante, ayant effrayé les chanoines par ses innovations musicales. Se tournant dès lors vers la musique profane, ce n'est qu'en 1804 qu'il obtint la direction de la Chapelle impériale et revint à la musique religieuse. Sur les débuts de sa carrière, Jean MONGRÉDIEN, Jean-François Le Sueur, op. cit., p. $15-204$.

(31) BNF, Musique, Lettres autographes, vol. 71, 209. Il est amusant de constater que Martini y souligne le rôle que joue le surintendant lors des cérémonies religieuses extraordinaires, « depuis Lully », tout comme Le Sueur à la même époque (Ibidem, vol. 67, p. 135, lettre au roi, 26 mai 1814).

(32) Ibid., 208, lettre à Lefèvre, 5 octobre 1814, demandant « de copier le plutôt possible la partition [d'un] pseaume, même les parties séparées [car] il se pourroit que celui-ci soit exécuté le premier ».

(33) Adélaïde de Place, La vie musicale en France au temps de la Révolution, Paris, Fayard, 1989, p. 48-49.

(34) $\mathrm{AN}, \mathrm{O}^{3} 282$, plusieurs échanges entre le baron de La Ferté, intendant des Menus Plaisirs, et le comte de Pradel, directeur général de la Maison du roi (lors de la seconde Restauration, il a remplacé Blacas sans avoir le titre de ministre), à propos des irrégularités de Martini qui ordonnance des états de paiement sans y être autorisé. 
auraient échafaudé de nouveaux projets ${ }^{35}$. De fait, Métoyen, bassoniste à partir de 1760 et doyen de la Musique du roi, qui semble au cour des revendications de l'ancienne chapelle, appartient à ces deux groupes ${ }^{36}$. Jadis chargé de dresser chaque année un état de la Musique (parfois illustré, nous l'avons vu), il en a conservé les minutes, sur lesquelles il s'appuie pour dresser des listes de ses anciens collègues, assorties de commentaires sur l'organisation générale, aussi bien que sur les capacités ou les adresses des survivants ${ }^{37}$. L'une d'elles évoque les musiciens « qui ont droit à être replacés ${ }^{38}$. Métoyen s'est donc investi pour ses anciens camarades, parmi lesquels figure son fils Guy Joseph, sobrement qualifié de « bon violoncelle » - quant à lui, trop âgé pour exercer la musique, il réclame, outre sa pension, «le titre honorifique de Bibliothécaire de la Grande Chapelle » ${ }^{39}$. Notons que ces documents ne précisent jamais l'appartenance de certains anciens à la Chapelle impériale et que les observations s'inscrivent toutes dans le registre le plus positif : « il chante avec beaucoup de goût » ou « très bien », voire « il fait encore plaisir ».

Métoyen pourrait être également à l'origine (ainsi que Martini, qui n'y apparaît pas) de deux listes intitulées « Projet d'organisation de la Musique du Roi », égarées au sein du fonds de la Musique du roi de l'Ancien Régime, mais indéniablement datables de $1814^{40}$. On y trouve des propositions d'emplois de musiciens présents à Paris, avec, pour certains d'entre eux, la précision « de l'ancienne chapelle » (royale, donc) ou « de la nouvelle chapelle » (i.e. impériale). Une autre main (qui pourrait appartenir

(35) «Lettre sur la Musique... », op. cit., p. 2-3.

(36) Hervé AudÉON et Cécile DAVY-RigAUX, « Jean-Baptiste Métoyen (1733-1822). Parcours et œuvre d'un musicien de la Chapelle royale, de l'Ancien Régime au début de la Restauration », Revue de musicologie, t. $94,2008, \mathrm{n}^{\circ} 2$, p. 347-385.

(37) $\mathrm{AN}, \mathrm{O}^{3} 375, \mathrm{n}^{\circ} 1$, Etat de la Musique du Roi dans les dernières années du Règne de Louis XVI ; Bibliothèque-musée de 1'Opéra de Paris, Arch. Div 14 [7 : Etat des sujets composant la Musique de la Chapelle du Roy, lors de sa destruction au 10 Aoust 1792, qui précise les dates d'entrée en service, les personnes décédées et le domicile des survivants ; mais aussi Ibidem, Liste des Musiciens de la Chapelle du Roi, qui restent éxistants en 1814. suivant leurs réceptions et le tems de leurs services jusqu'en 1792. pour faire connoître ceux qui ont droit à la totalité de leurs vétérances, ou assez jeunes pour y être replacés (autre version, avec quelques différences minimes : Bibliothèque-musée de l'Opéra de Paris, Arch. Div 14 [1); Etat nominatif des anciens Musiciens de la Chapelle du Roi encore existant, qui précise l'âge (systématiquement abaissé d'environ cinq ans, mais Métoyen ne dispose pas des extraits de baptême de ses anciens collègues), l'année de réception, le montant du traitement en 1792, et résume les capacités musicales de chacun.

(38) Ibid., Liste des Musiciens de la Chapelle du Roi, qui restent existans en 1814, et qui ont droit à être replacés. Trente-trois noms cités, « dont sept sont à la Chapelle du Roi ». Une autre main a tracé des croix, barré certains noms et ajouté un commentaire pour Ertault : « mauvaise réput. ».

(39) Ibid., Extraits des Réclamations..., n ${ }^{\circ} 5$; Extraits des Placets en demande de pensions, $\mathrm{n}^{\circ} 2$. Le titre convoité est accordé à compter du $1^{\text {er }}$ janvier 1815.

(40) $\mathrm{AN}, \mathrm{O}^{1} 842, \mathrm{n}^{\circ} 104$ et $\mathrm{n}^{\circ} 105$. 
à Le Sueur) a ajouté des commentaires, parfois laudateurs (« $1^{\mathrm{er}}$ talent », « fort en état de servir », comme Cardon), d'autres fois interrogatifs (« bien âgé pour chanter » pour Platel ou Josephini, à propos de qui on lit en outre «a-t-il conservé ses moyens ? ), plus rarement assassins («n’est pas musicien » pour Catalan, qui, comme par hasard, est « proposé par Martini », ou pire, sur Théodore : « il n'est nullement musicien et ne chante ni juste ni en mesure »).

Tout ceci paraît loin de la tentative de putsch évoquée par Le Sueur, lui-même du reste plus nuancé et conciliant dans certains de ses écrits. Au demeurant, bien des anciens ne sont plus en mesure de servir et ne se font aucune illusion à ce propos. Une comparaison entre les listes des trente-neuf musiciens encore vivants et les suppliques adressées à l'administration de la Maison du roi ${ }^{41}$ montre deux choses. En premier lieu, ceux qui ont dû reconstruire leur vie après la dissolution de la Musique du roi ne sont pas toujours désireux de reprendre du service. Ainsi, Métoyen fils, bien que réintégré au début de 1815 , envoie une lettre de démission fort bien tournée à Martini, dans laquelle il argue « d'une Place que j' occupe ne me laissant pas la liberté de remplir exactement le devoir que je m'imposerais en acceptant celle que vous et Monseigneur le Duc de Fleury avez cru juste de me rendre à la Musique du Roi $»^{42}$. Quant aux autres, ils aspirent plus à une retraite, assortie d'une pension conforme à l'édit de 1782, qu'à une réintégration. Cette catégorie, la plus importante, regroupe les vétérans et ceux qui auraient dû l'être sans la Révolution. Ainsi, l'ancien hautboïste Jean-François Rostenne, vétéran depuis 1782, souhaite retrouver la totalité de sa pension, avançant ses déboires, à Paris et à Lyon, comme gage de sa fidélité inébranlable à la monarchie ${ }^{43}$. Jean Joseph Évelart, ayant servi 16 ans (de 1777 à 1792) comme flûtiste, « reparoit » ${ }^{44}$ pour réclamer la pension égale à la totalité de ses appointements, « d'après l'édit du Roi de 1782, qui [1']accorde aux instrumens à vent, après 15 années de service [...] Sa Majesté n'ayant pas abrogé l'édit sus-nommé $»^{45}$.

Certes, comme l'écrit Le Sueur, la plupart des « anciens musiciens de Louis XV et de Louis XVI [...] ont de 66 à 75 ans » ${ }^{46}$ et il est à

(41) $\mathrm{AN}, \mathrm{O}^{3} 375$, une vingtaine de réclamations.

(42) Ibidem, lettre du 3 janvier 1815.

(43) Ibid., n 14.

(44) Ibid., Liste des Musiciens [...] qui ont droit à être replacés.

(45) Ibid., lettre du 3 février 1815.

(46) Ibid., Direction provisoire. Le Sueur noircit la réalité : seuls onze anciens sont âgés de plus de 66 ans (dont Martini, 73 ans, et Métoyen, 81 !), mais il est vrai que plus de la moitié sont sexagénaires et que sept seulement ont moins de 50 ans, dont Kreutzer, alors en place. 
craindre qu'ils ne réclament des places que pour obtenir une pension tenant compte des services passés. Pourtant, ces neuf mois de projets et de contreprojets accouchent d'une organisation assez peu bouleversée, malgré des mouvements de personnels non négligeables.

\section{L'établissement de la Musique de la Chapelle du roi (janvier 1815)}

Le $1^{\text {er }}$ janvier 1815, la Musique est définitivement organisée avec un effectif de cent personnes (dont quatre-vingt huit musiciens) ${ }^{47}$ : quaranteneuf nouveaux venus émargent désormais sur les listes, tandis que cinquantesix membres de la Musique provisoire sont remerciés. C'est donc un échec pour Le Sueur, qui sauve sa place, mais (exemple typique de la méthode de distribution des places sous la première Restauration) doit la partager avec Martini et perd une partie de son traitement, ramené de 10000 à 6000 francs.

L'une des décisions les plus marquantes confirme les craintes exprimées par Le Sueur six mois plus tôt : les chanteuses sont exclues de la Musique, remplacées par des «pages » (qui s'ajoutent aux cent personnes de l'état). Ces dix jeunes garçons (plus deux suppléants, ce qui correspond à la situation de 1790), placés sous la houlette du pianiste Louis Emmanuel Jadin, lui-même ancien page de la Musique du roi, sont censés assurer désormais le pupitre de dessus, avec l'aide du castrat Josephini, dont la voix semble encore solide à 60 ans passés, et de quatre nouveaux faussets ${ }^{48}$. L'éviction des femmes d'un ensemble voué spécifiquement à la musique d'église sonne comme un écho aux débats sur le sujet qui avaient agité la Chapelle royale sous le règne de Louis XV : alors que son prédécesseur avait autorisé l'apparition de quelques femmes à la tribune de sa chapelle, elles en furent écartées au profit de nouveaux castrats italiens.

Vingt anciens surnagent parmi les nouveaux venus de 1815 (41\%), dont Mornard, qui retrouve sa place de secrétaire. Contrairement aux

(47) $\mathrm{AN}, \mathrm{O}^{3} 296, \mathrm{n}^{\circ} 502$, Personnel de la musique de la chapelle du Roi. Appointemens de Janvier $1815 ; \mathrm{O}^{3} 375$, grande liste alphabétique, sans titre, avec renvois aux numéros d'ordre du document précédent, lieux et dates de naissance, services rendus sous Louis XVI et « sous le dernier gouvernement » (expression qui remplace celle, biffée, de « Musique de Buonaparte »), et date d'admission dans la Musique de Louis XVIII. Les employés non musiciens sont le facteur d'orgue, l'accordeur, deux garçons de la musique, un porteur d'instruments (ces deux fonctions étaient réunies auparavant), deux avertisseurs, un secrétaire, un bibliothécaire et, pour les pages, un garçon, un précepteur et un maître d'écriture. Plusieurs brevets, datés pour la plupart du 27 décembre 1814, en $\mathrm{AN}, \mathrm{O}^{3} 375$.

(48) Leurs antécédents musicaux sont inconnus. L'un d'eux, Ducroc, a été auparavant vérificateur à la cour des comptes pendant 28 ans $\left(\mathrm{AN}, \mathrm{O}^{3} 354, \mathrm{n}^{\circ} 324\right.$, demande de pension par sa veuve). Il pourrait être le fils d'un ancien musicien du roi. 
craintes de Le Sueur, la Musique n'a pas été assaillie par des vieillards cacochymes chasseurs de pension. Deux septuagénaires qui ne peuvent plus chanter, Le Roux (qui avait repris du service en 1802) et Pusseneau, font figures d'exception en retrouvant leurs places de 1792, comme avertisseurs, en charge de prévenir leurs collègues des activités musicales ${ }^{49}$. Les autres sont tous opérationnels, qu'ils soient sexagénaires, comme la basse Platel, les ténors Richer et Guichard (également maître de chant des pages), le corniste La Motte, le hautboïste Garnier ou le violoniste Guénin, ayant servi Charles IV d'Espagne exilé à Marseille ${ }^{50}$, ou quadragénaires, comme les violonistes Marcou et Eigenschenck, le contrebassiste Gelinek, le flûtiste Besozzi ou deux anciens pages, désormais ténors, Félix et Durais ${ }^{51}$. Les autres sont des hommes nouveaux, jeunes pour la plupart, dont la moitié recrutés comme chanteurs. Leurs carrières antérieures sont quasi inconnues ${ }^{52}$, mais tous semblent avoir été reçus pour leurs qualités musicales - leur longévité à la Chapelle milite en ce sens -, y compris Cauchoix, fils d'un ancien musicien du roi. Pas de favoritisme inconsidéré, ni de dévalorisation de la qualité musicale, donc ${ }^{53}$. Une faveur est cependant accordée aux «revenants »: ils ont droit à un traitement annuel de 1500 francs, quand leurs collègues du même pupitre touchent 300 ou 400 francs de moins ${ }^{54}$.

Quant aux réformés, près des deux tiers (34 personnes, dont 15 femmes) sont des chanteurs. Plus qu'une épuration politique - sauf, peutêtre, pour Lays dont les opinions révolutionnaires étaient notoires ${ }^{55}-$, c'est un nettoyage moral : la défense présentée par Le Sueur n'a pas su détruire l'idée que des chanteurs d'opéra étaient inconvenants à la chapelle. La précision de n'avoir jamais été attachée au théâtre que Mme de Staiti a cru

(49) Le surintendant envoie des billets manuscrits pour les répétitions : deux exemples, signés Martini, du 28 décembre 1814, pour la répétition générale de la messe du $1^{\mathrm{er}}$ janvier 1815, « qui aura lieu le Vendredy $30 \mathrm{X}^{\text {bre }}$, a la chapelle, a midy $\frac{1}{2}$ » (BNF, Musique, Lettres autographes, vol. 71, 205 ; Bibliothèque-musée de l'Opéra de Paris, L.A.S. Martini).

(50) Claire LAmQueT, « Marie-Alexandre Guénin (1744-1835), un compositeur entre Lumières et romantisme », Dix-huitième siècle, $\mathrm{n}^{\circ}$ 43, 2011, p. 136.

(51) Son père, basse-taille, fut représentant des musiciens du roi à l'assemblée du bailliage de Versailles en 1789. En juillet 1815, Durais prend aussi en charge le secrétariat à la place de Mornard, nommé inspecteur.

(52) Deux militaires (Morena et Consul, ayant aussi servi au théâtre italien), un maître de piano et de chant de la maîtrise de Notre-Dame (Cornu) (AN, $\mathrm{O}^{3} 354$, états de services, 1825).

(53) La nomination de Chevalier comme garçon de la musique, récompense de l'aide qu'il a apportée au duc de Duras pour émigrer, n'a aucune incidence musicale (AN, $\mathrm{O}^{3} 354$ ).

(54) Cette différence ne saute pas aux yeux sur les états de traitements mais les ajustements des premiers temps, qui entraînent le remplacement des anciens démissionnaires par des nouveaux, obligent à revoir à la baisse les appointements, d'où plusieurs courriers en ce sens $\left(\mathrm{AN}, \mathrm{O}^{3} 282\right.$, Rohan à Blacas, 24 et 28 février 1815 , et rapport sur le sujet).

(55) Adélaïde DE PLACE, op. cit., p. 129. 
devoir apporter dans sa réclamation du 11 janvier 1815 est symptomatique à cet égard ${ }^{56}$. L'éviction du deuxième chef d'orchestre, Rochefort, qui occupe un poste similaire à l'Opéra, découle sans doute du même principe, mais aussi d'une volonté de rationalisation : Persuis, désormais « chef de chœur », suffit d'autant mieux à la tâche que Kreutzer peut le suppléer. Il est plus difficile de comprendre la réforme de cinq violoncellistes et d'autant de seconds violons, si ce n'est pour Ertault, de l'ancienne Chapelle, qui est mis à la retraite ${ }^{57}$.

Bien entendu, les réclamations ne tardent guère. Marand, « $2^{\mathrm{e}}$ secrétaire de la Direction », prétend, avec une impudence rare et une démonstration aussi douteuse qu'alambiquée, avoir servi le roi depuis onze ans. La succession des rois Bourbons étant ininterrompue depuis Louis XVI, écrit-il, « il n'y a point d'interrègne. Cet argument est péremptoire et sans réplique. Mes onze années de service ont donc incontestablement eu lieu dans la musique de la Maison du Roi. Je suis donc apte à jouir du Bénéfice de l'Edit de $1782 » .$. Et de réclamer sa pension entière, en insistant sur le fait qu'il n'a pas démissionné ${ }^{58}$. Cet aplomb insolent demeure néanmoins rare $^{59}$, face aux suppliques larmoyantes des « pauvres refformez de la chapelle », qui implorent le paiement des neuf mois de service de 1814 : «ils attendent cette grace de votre humanité si vous etiéz persuadéz combien ils gémissent vous en seriez touché ${ }^{60}$. Dans une autre du même tonneau, datée du 15 mars, l'auteur, malheureusement anonyme, ne craint pas d'affirmer être prêt à « partir pour la défense du roi » - à cette date, Napoléon est déjà à Autun -, mais ne pouvoir le faire tant que ses neuf mois de service pour le roi ne lui auront pas été payés. Il a en effet « besoin de ce secours pour le laisser a [sa] famille pour la soulager pendant [son] absence » et n'hésite pas à finir ainsi : « plusieurs voudroient se jetter aux pieds du roi pour implorer sa miséricorde ! $»^{61}$. Gageons qu'il n'en fit rien et fut réintégré à compter du 20 mars, comme la quasi-totalité des réformés de janvier, dans la Musique impériale ressuscitée.

(56) $\mathrm{AN}, \mathrm{O}^{3} 375, \mathrm{n}^{\circ} 9$.

(57) Ibidem, $\mathrm{n}^{\circ} 13$. Ertault semble surpris par cette décision (imputable à sa «mauvaise réput[ation] », signalée plus haut ?) et met en avant ses services militaires, à l'Opéra et à la Chapelle pour obtenir une pension complète.

(58) Ibid., $\mathrm{n}^{\circ} 5$.

(59) Autre exemple, moins outré, mais avec les mêmes arguments pour le bassoniste Dossion (Ibid., $\left.\mathrm{n}^{\circ} 15\right)$

(60) $\mathrm{AN}, \mathrm{O}^{3} 282$, ? [signature illisible] à Blacas, 8 mars 1815.

(61) Ibidem, lettre non signée, 15 mars 1815. 


\section{Le sursaut des Cent-Jours et la stabilisation}

Les états de paiement du « gouvernement intérimaire ${ }^{62}$ disent clairement le retour à l'organisation de 1814 : à neuf exceptions près (dont Séjan), tous les musiciens de la Musique provisoire y sont présents. En revanche, aucun nouveau de janvier n'y apparaît et ce n'est vraisemblablement pas par choix de leur part. Bien entendu, aucun n'accompagne Louis XVIII à Gand ${ }^{63}$ et Martini ne tarde pas à informer le comte ÉlisabethPierre de Montesquiou, grand chambellan de Napoléon, que « les musiciens employés à la chapelle depuis le $1^{\text {er }}$ janvier 1815 , et qui ne font plus partie de la musique de la Chapelle de S.M. » demandent à recevoir au plus vite leurs appointements du mois de mars ${ }^{64}$. Dans ce groupe d'une quarantaine se rencontrent certains des neuf disparus évoqués plus haut : est-ce l'expression d'un choix réfléchi ? C'est vraisemblable pour les ténors Courtin et Rignault qui, dans l'espoir d'accélérer le paiement d'une indemnité due pour 1814, soulignent n'avoir point servi « pendant l'interregne » et avoir « été privé [s] de leur traitement pendant trois mois ${ }^{65}$. Quant à Lhoste qui avait chanté sous Louis XVI puis dès 1802, son décès, survenu le 19 avril 1815 «par suite de la douleur qu'il éprouva à la révolution du 20 mars précédent », est cause de son absence... à croire du moins sa veuve et ses deux fils ${ }^{66}$. De là à y lire l'aboutissement dramatique d'un choix en faveur des Bourbons, il y a un pas que je me garderai bien de franchir.

Le tourbillon des Cent-Jours n'a guère été propice aux activités musicales, ce qui entraîne la mise en paiement de tous les traitements... après le retour de Louis XVIII. Les états dressés pour les trois mois « d'interrègne » ne comptabilisent que les écartés, considérés comme des

(62) AN, $\mathrm{O}^{2}$ 62, Maison de S.M. l'Empereur. Exercice 1815, 10 derniers jours de mars, avril, may.

(63) Dans un état de ses services établi en 1825, le chanteur Huet affirme avoir suivi le roi à Gand, mais il était alors officier de santé, pas encore musicien (AN, $\left.\mathrm{O}^{3} 354\right)$.

(64) $\mathrm{AN}, \mathrm{O}^{3} 282$, lettre de Montesquiou au ministre des Finances, $1^{\mathrm{er}}$ avril 1815. Ces musiciens n'ont pas été entendus, si l'on en croit l'interrogation de l'administration royale sur l'opportunité de payer Mme Camporesi «pour services dans la Maison de Buonaparte [...] d'autant [...] que ce dernier n'a fait acquitter aucun des traitemens dus pour la Maison du Roi, quoique les états fussent ordonnancés et que des fonds suffisans aient été laissés au trésor au Départ de S.M. » (Ibid., rapport du 26 octobre 1815).

(65) $\mathrm{AN}, \mathrm{O}^{3} 285$, lettre à Pradelle [sic], s.d. [octobre 1815]. Rignault, entré dès 1802, déclare pourtant en 1825 n'avoir servi que « trois années [...] sans appointemens » sous l'Empire, déclaration qui semble attester un rejet viscéral de cette période ( $\left.\mathrm{AN}, \mathrm{O}^{3} 354\right)$.

(66) Ibidem, supplique à Pradel, 24 octobre 1815. Un mois et demi plus tard, l'un des fils demande une survivance de basse, qui lui est refusée, car « S.M. ne veut plus que ce mode soit suivi pour les places de Sa Maison ». On l'encourage à passer une audition en cas de vacance $\left(\mathrm{O}^{3 *} 279\right.$, p. $47, \mathrm{n}^{\circ} 169$, réponse du 11 décembre 1815$)$. 
fidèles des Bourbons et bien entendu tous réintégrés en juillet $1815^{67}$. Pour autant, il ne s'agit pas simplement de refermer une parenthèse en revenant à la situation de mars.

L'effectif global de la seconde Restauration, désormais de cent-huit personnes (sans les pages), accuse une augmentation de $8 \%$, dont le retour de chanteuses constitue le facteur le plus voyant.

Un an plus tôt, Le Sueur soulignait que, au service funèbre célébré à Notre-Dame pour les Bourbons morts pendant la Révolution, le 14 mai 1814, «l'execution [du Requiem de Jommelli] a été mauvaise, parce qu'on avait défendu que les femmes y fussent et qu'elles y ont été remplacées par les mêmes enfants qu'on admettra pour être pages $»^{68}$. La courte expérience des messes du début de 1815 a sans doute abouti au même constat. C'est pourquoi, dès le 18 juillet, Le Sueur, Martini, Cherubini et Plantade $^{69}$ écrivent ensemble au duc de Rohan pour réclamer quelques chanteurs des deux sexes, tirés « des premiers théâtres de la capitale $»^{70}$. Les quatre hommes, dont les deux premiers parlent probablement pour la première fois d'une même voix, obtiennent gain de cause, avec le recrutement de cinq « récitantes », chargées des solos : deux anciennes «premières cantatrices » de la Chapelle impériale (Mmes Armand et Albert-Himm) et trois nouvelles recrues, dont Mme Lemonnier-Regnault, future gloire de l'Opéra-comique. Les pages ne sont pas supprimés pour autant. Le Sueur insistait d'ailleurs, dès avant 1814, sur l'utilité de tels lieux de formation musicale qui, avec les maîtrises de cathédrales peu à peu recréées en France, constituaient la pépinière des voix masculines à venir. Réduits au nombre de six, plus deux suppléants, ce n'est qu'à la fin de l'année que ces jeunes garçons sont installés dans un pensionnat à la charge de Jadin, après un temps d'improvisation qui avait entraîné quelques plaintes des parents ${ }^{71}$.

L'adoucissement des mesures d'exclusion de janvier ne concerne pas uniquement les deux chanteuses évoquées plus haut, puisque trois anciens chanteurs (Chenard et Martin, récitants, et Prévost, choriste) les rejoignent,

(67) AN, $\mathrm{O}^{3}$ 379, Maison du Roi, Exercice 1815. Mois de Mars, avec une partie portée « pour vingt jours », puis Mois d'Avril, Mai et Juin.

(68) $\mathrm{AN}, \mathrm{O}^{3} 375$, Direction provisoire.

(69) Cherubini avait été nommé surintendant en survivance de Martini dès janvier 1815 et Plantade, qui était page dans les années 1770, remplace Persuis, avec le titre nouveau de maître de chapelle.

(70) $\mathrm{AN}, \mathrm{O}^{3} 291$, lettre publiée dans Marius VACHON, « La Chapelle royale et le corps des pages de la Musique de 1815 à $1830 »$, Le Ménestrel, 1880, 46 ${ }^{\mathrm{e}}$ année, $\mathrm{n}^{\circ} 22$, p. 169-170:170.

(71) $\mathrm{AN}, \mathrm{O}^{3} 282$, arrêté du duc de Rohan, du 30 août 1815 , et différents mémoires ; $\mathrm{O}^{3 *} 279$, : p. 169-170: 170, p. 37-38, n 126 , lettre à Jadin du 7 novembre 1815. 
ainsi que le clarinettiste Lefèvre, le corniste Vandenbrock, le violoniste Cartier et deux violoncellistes, Boulanger et Charles.

D’un autre côté, 46 des révocations de janvier sont confirmées, et même renforcées par une dizaine de personnes ayant pourtant été titularisées en janvier 1815. Parmi ces dernières, le départ le plus marquant est celui de Persuis, souvent expliqué par la reprise, pendant les Cent-Jours, de son Triomphe de Trajan, donné sur la scène de l'Opéra dès le 26 mars et joué cinq fois... cinq fois de trop ${ }^{72}$. Les autres exclus, moins exposés, restent peut-être sur la touche pour avoir trop tardé à se manifester. De fait, loin d'être automatiques, les réintégrations font l'objet de demandes des intéressés, ainsi qu'il ressort des rejets de réclamations arrivées trop tard. Ainsi, Murgeon, fausset de Louis XVI puis de la Chapelle impériale, attend le 20 décembre 1815 pour demander, avec le soutien de Le Sueur, à réintégrer sa place, et se voit répondre que, « le travail étant terminé et approuvé par le Roi, il est impossible d'y apporter aucun changement ${ }^{73}$. Ces délais, généralement méconnus des musiciens, expliquent que les recalés de juillet 1815 reviennent parfois plus tard dans les rangs de la Chapelle, au gré des départs et des concours de remplacement. C'est le cas du hautboïste Vogt, du ténor Bouffet, des basses Devilliers et Lecocq, de la mezzo Staiti et même du fausset Théodore, pourtant cible d'une note cruelle en $1814^{74}$.

Enfin, la réorganisation de juillet 1815 permet de pensionner quelques artistes trop âgés pour servir, dont certains anciens qui ne reparaissent sur les états que pour être placés en retraite. C'est le cas du flûtiste Évelart, évoqué plus haut, dont la brève apparition en tant que « $1^{\text {er }}$ hautbois », sur l'état de juillet 1815 , vaut marchepied pour la retraite ${ }^{75}$. Le paiement de ces pensions, qui avait été un casse-tête, source de dépassements sous l'Ancien Régime, avait été largement amélioré sous l'Empire par la création d'une « caisse de vétérance » alimentée par la retenue de $3 \%$ des traitements. Ce système, prorogé par l'ordonnance du 3 décembre 1814,

(72) Jean MONGRÉDIEN, La musique en France, op. cit., p. 57-59 ; David CHAILlOU, Napoléon et l'Opéra. La politique sur la scène 1810-1815, Paris, Fayard, 2004, p. 83-85 et 522-524 pour les représentations. en 1818).

(73) $\mathrm{AN}, \mathrm{O}^{3 *} 279$, p. $51, \mathrm{n}^{\circ} 188,25$ décembre 1815 , réponse à Murgeon (finalement réintégré

(74) $\mathrm{AN}, \mathrm{O}^{1} 842, \mathrm{n}^{\circ} 105$, cité supra.

(75) $\mathrm{AN}, \mathrm{O}^{3} 282, \mathrm{n}^{\circ} 154$, Etat nominatif des Musiciens et Employés de la Chapelle du Roi aux quels Mr le Duc de Rohan a accordé des Gratifications et Secours pour le Service quils ont fait pendant 1815, avec les musiciens réformés à la fin. Il y côtoie le corniste Collin, le pianiste Piccini, la basse Fontaine, les bassonistes Henry et Judas, les clarinettistes Dacosta et Solere, ainsi que ses anciens collègues Richer et Eigenschenck. 
permit de dédommager les vétérans de l'Ancien Régime aussi bien que ceux qui avaient perdu leur emploi à la chute de l'Empire, « à condition toutefois qu'ils aient servi Napoléon à partir du $1^{\text {er }}$ janvier $1810 »^{76}$.

Le 14 février 1816, le décès de Martini ${ }^{77}$ libère la place pour son survivancier Cherubini et permet de fixer l'organisation de la Musique jusqu'en juillet 1830, par un règlement daté du 27 février $^{78}$. Le résultat le plus tangible de ce règlement, qui établit un roulement trimestriel des surintendants, est l'exécution alternée, au cours de la messe, des compositions de Le Sueur et de Cherubini, en plus « des ouvrages les plus renommés des anciens compositeurs ». Ce texte, truffé de réminiscences des règlements de 1761 et 1782, peut être lu comme la victoire de l'Ancien Régime, mais il est surtout le témoin d'une volonté d'efficacité qui s'inscrit dans la continuité du fonctionnement progressivement mis en œuvre par Le Sueur depuis 1804.

En définitive, malgré un renouvellement de la moitié de son effectif, la Musique de la Chapelle du roi ressemble fort à celle de l'empereur. Certes, environ $20 \%$ du personnel de 1816 est constitué de musiciens de l'Ancien Régime (donc cinq qui étaient pages), mais plus de la moitié des artistes exerçaient déjà sous l'Empire. Les fantômes de l'Ancien Régime ne s'expriment donc pas tant à travers les réapparitions de ceux « de l'ancienne chapelle », du reste parfois fugaces (Martini, Métoyen père et fils... et d'autres mis à la retraite), que par des choix terminologiques (réapparition du titre de surintendant, distinction entre les voix de hautecontre et de taille, regroupées sous l'appellation ténor pendant l'Empire) ou structurels (éviction des femmes, retour des pages), qui s'avèrent finalement de peu de portée et pas toujours durables. Ainsi, le recours aux chanteuses ne cesse de progresser au fil du temps : en 1829, ayant totalement évincé les hommes (qui sont encore quatre en $1826^{79}$ ), une vingtaine de femmes

(76) Philip MANSEL, La Cour...op. cit., p. 123-124.

(77) $\mathrm{AN}, \mathrm{O}^{3} 379$, acte de décès, 15 février 1816, sur déclaration faite par Plantade.

(78) $\mathrm{AN}, \mathrm{O}^{3} 290$. S'y trouve également l'ordonnance sur la Musique du 13 mars 1830 (avec le rapport préparatoire de La Ferté) qui n'a pas été appliquée, la suppression de la Chapelle du roi ayant suivi le changement de régime.

(79) Ibidem, Etat général de la Musique du Roi, s.d. [1826]. Parmi les quatre dessus masculins, Josephini et Murgeon achèvent, par une retraite fixée au $1^{\mathrm{er}}$ janvier 1826, une longue carrière commencée sous l'Ancien Régime. Le premier, arrivé en France en 1774, est non seulement le dernier castrat à s'être produit en France, mais aussi le seul musicien qui ait chanté aux sacres de Louis XVI et de Charles X. 
peuplent les deux pupitres de dessus d'un chœur désormais à quatre voix, au sein duquel les ténors ont définitivement supplanté les hautes-contre ${ }^{80}$.

Si, comme avant 1792, l'activité religieuse apparaît prépondérante, ne serait-ce que dans l'appellation retenue pour le corps de musique, c'est en fait le résultat d'un choix effectué sous le Consulat, lors de la refondation d'une Chapelle, conséquence du concordat autant que des tendances monarchiques du premier consul Bonaparte. L'activité musicale y est désormais bien éloignée de celle qui avait cours jusque dans les derniers temps de l'Ancien Régime : le règne du motet à grand chœur, déjà critiqué au siècle précédent comme un héritage obsolète du Roi-Soleil, est clos, tandis que la messe quotidienne n'est plus qu'un souvenir. Les deux derniers Bourbons ne remettent pas en cause cette évolution, dont Le Sueur est le principal artisan. Néanmoins, la messe impériale, puis royale, célébrée le dimanche en présence du souverain dans la chapelle des Tuileries, se distingue toujours nettement de la pratique des grandes paroisses et des cathédrales de France. Comme à l'époque versaillaise dont elle s'inspire, elle prend l'aspect d'une messe basse accompagnée de musique. Ce que Le Sueur nomme «messe » revêt des formes diverses, qui l'apparentent à l'oratorio, au motet ou au fragment de messe. En dehors des fêtes religieuses particulières, solennisées par l'exécution d'une « messe solennelle » constituée de l'ordinaire complet mis en musique, Louis XVIII, comme son aïeul et comme son défunt frère, assiste à l'office depuis la tribune où ne lui parviennent pas les paroles du prêtre, couvertes par la musique. L'ensemble ne dure qu'une demi-heure et se clôt traditionnellement par le chant du Domine, salvum fac regem. La similitude de la forme autant que de la durée n'a guère reçu d'écho parmi les contemporains. Qui se souvient de la messe de l'ancienne monarchie ? D'ailleurs, l'acoustique sourde de la chapelle des Tuileries ne peut rivaliser avec celle de Versailles. Et surtout, la musique, dont le style plus théâtral que religieux a tant surpris les étrangers, n'est guère comparable aux compositions de Delalande, de Blanchard ou de Giroust 
qui, jusqu'en octobre 1789, retentissaient quotidiennement sous la voûte peinte de la chapelle de Versailles ${ }^{81}$.

Youri CARBONNIER

CREHS - Université d'Artois

9, rue du Temple BP 10665

62030 Arras cedex

youri.carbonnier@univ-artois.fr

(81) Jean MONGRÉDIEN, La musique en France, op. cit., p. 164-183. 